\title{
LOS IMPACTOS DEL EWOM EN HOTELES
}

Salvi, Fabiana ${ }^{1}$

Serra Cantallops, Antoni ${ }^{2}$

Ramón Cardona, José 3

Universitat de les Illes Balears

fabiana salvi@hotmail.com

antoni.serra@uib.es

jose.ramon@uib.es

Material original autorizado para su primera publicación en la revista académica REDMARKA. Revista Digital de Marketing Aplicado.

https://doi.org/10.17979/redma.2013.02.010.4765

Recibido: 10 Marzo 2013

Aceptado 18 Junio 2013

\section{RESUMEN}

El objetivo de este trabajo es la revisión de las investigaciones existentes referente a los impactos del electronic Word-of-Mouth (eWOM) dentro del sector de los hoteles. La revisión se centra en los artículos publicados durante los últimos años en revistas académicas especializadas en turismo y de gran relevancia internacional. Como resultado de esta revisión se identificaron los

\footnotetext{
${ }^{1}$ Doctoranda en Economía de la Empresa, Universidad de las Islas Baleares (UIB). Licenciada en Administración de Empresas por la Universidade Luterana do Brasil (ULBRA). MBA Internacional en Gestión para el Desarrollo Sostenible por la Universidade Luterana do Brasil (ULBRA), en convenio con Fondation Universitaire Luxembourgeoise (FUL), Bélgica. Máster en Gestión Turística (MTA) por la Universidad de las Islas Baleares (UIB).

${ }^{2}$ Associate Professor. Marketing and Market Research Area. Business Economics Department. University of the Balearic Islands.

${ }^{3}$ Licenciado en Administración y Dirección de Empresas, Licenciado en Economía y Doctor en Economía de la Empresa por la Universidad de las Islas Baleares. Profesor asociado de Comercialización e Investigación de Mercados en la Universidad de les Illes Balears. Colaborador del grupo Dirección y Gestión de Empresas y Destinos Turísticos.
} 
principales impactos generados por el eWOM desde la perspectiva de los consumidores y desde la perspectiva de las empresas. Para los consumidores, los comentarios poseen efectos significativos en la toma de decisiones, al facilitar el acceso y procesado de la información, y en la reducción del riesgo de compra de un producto intangible como el turismo. Para las empresas, puede generar oportunidades y amenazas para su imagen, como también influir en el volumen de ventas y en la generación de lealtad. Este trabajo pretende clarificar e informar sobre el estado de la cuestión de cara a futuros estudios relacionados con el eWOM y los hoteles.

Palabras Clave: hoteles, eWOM, revisión.

\section{ABSTRACT}

\section{THE IMPACTS OF EWOM IN HOTELS}

The objective of this study is the review of the existing literature relating to the impacts of the electronic Word-of-Mouth (eWOM) within the hotel sector. The review focuses on published articles during the last years in academic recognized journals specialized in tourism and of great international importance. As a result of this review we have identified the main impacts generated by the eWOM from a consumer perspective and from a business perspective. For consumers, the comments have significant decision-making purposes, to facilitate access and processing of information, and on reducing the risk of buying an intangible product as tourism. For businesses, it generates opportunities and threats to its image, the volume of sales and the generation of loyalty. This study aims to clarify and inform about the State of the art with regard to future studies related to eWOM and hotels.

Key Words: hotels, eWOM, review. 


\section{INTRODUCCIÓN}

El avance de las tecnologías de la información y la introducción de nuevas formas de comunicación, tanto entre consumidores como entre empresas y consumidores, genera impactos importantes en las estrategias de marketing y los resultados empresariales, ya que necesitan constantemente adaptarse a los cambiantes comportamientos y procesos de decisión de los consumidores.

Los procesos de decisión de compra están condicionados por diversas variables que influyen en la elección del consumidor respecto a determinados productos y servicios. Los clientes pueden elegir un hotel por su ubicación (es decir, cerca del aeropuerto, zona turística o centro urbano), nombre de la marca, diversas instalaciones (piscina, campo de golf, spa y gimnasio), calidad de servicio, precio, programa de fidelización y valoraciones de calidad de huéspedes anteriores. Cualquiera de ellos o todos estos factores podrán formar parte de la elección del cliente (Verma, 2010). Otras variables, como ambiente y diseño del establecimiento, podrán agregarse al conjunto de variables.

En este sentido, uno de los factores que afectan en el proceso de decisión del consumidor es el Word-of-Mouth (WOM), definido por Harrison-Walker (2001) como "una comunicación informal, de persona a persona, entre un comunicador no comercial y un receptor, con respecto a una marca, un producto, una organización, o un servicio". Dickinger y Basu (1994) definen WOM como "una comunicación volitiva después de la compra por parte de los consumidores". Gran parte de los estudios analizan el WOM como un factor que en mayor o menor medida influye en los consumidores a la hora de la elección de productos y servicios. Yoon y Uysal (2005) consideran que WOM es "una de las fuentes de información más buscadas a menudo por las personas interesadas en viajar".

Electronic Word-of-Mouth (eWOM), también referido a menudo como online reviews, online recommendations $u$ online opinions, ha ganado en importancia con la aparición de nuevas herramientas tecnológicas. Litvin et al. (2008) definen eWOM como "todas las comunicaciones informales dirigidas a los consumidores mediante tecnologías basadas en Internet relacionadas con el uso o características de bienes y servicios, o de sus vendedores". 
Esta definición incluye la comunicación entre los vendedores y los consumidores, así como entre los propios consumidores. Su tipología es bidimensional: a) el alcance de la comunicación: de uno a uno (correos electrónicos), uno a muchos (portales de opinión) o muchos a muchos (comunidades virtuales); y b) el nivel de interactividad: del asincrónico (correo electrónico, portales de opinión, blogs) al síncrono (chats, foros, mensajería instantánea). Además, el eWOM ha demostrado tener una poderosa influencia sobre la selección o no selección de una marca (Casaló et al., 2010). Worthington et al. (2010) indican que los consumidores que son muy leales tienen mayor probabilidad de expresar positivamente el WOM para la marca y actuar como defensores de la misma.

Las principales diferencias entre WOM y eWOM pueden identificarse en el alcance de los comentarios (en términos de número de personas que pueden ser influenciadas) y la rapidez de interacción. Con respecto a esta comparación, Sun y Qu (2011) concluyen que "comparado con el tradicional WOM, el eWOM es más influyente debido a su velocidad, comodidad, alcance de uno a muchos, y la ausencia de la presión humana cara a cara". Schiffman y Kanuk (2000) describen las razones adicionales que atraen la atención de los consumidores, en relación al WOM y eWOM, de la siguiente manera: "La expectativa de recibir información que puede disminuir el tiempo de decisión y el esfuerzo y/o contribuir a alcanzar un resultado más satisfactorio en la decisión". Esta amplitud de alcance del eWOM y la facilidad en acceder a las valoraciones puede afectar profundamente los resultados de las empresas y por eso cada vez es más importante entender los factores generadores, las influencias y los impactos que resultan de su uso.

La industria del turismo se ve fuertemente afectada por el eWOM y, dentro de la industria del turismo, los hoteles son probablemente los más afectados. Basado en la creciente importancia de la reputación online derivada de los comentarios (que se indica en este artículo como eWOM), se propone en este estudio el análisis de la bibliografía académica existente sobre el concepto con la finalidad de recopilar y clarificar los aspectos relacionados a esta nueva 
forma de comunicación y su impacto en el comportamiento de los consumidores.

\section{METODOLOGÍA}

Para este estudio se han considerado los artículos publicados en los últimos años, sobre Word-of-Mouth (WOM) y electronic Word-of-Mouth (eWOM) relacionados con la industria hotelera. Se identificaron las publicaciones científicas en función de su relevancia, puntuación académica y volumen de artículos relacionados con el tema. Se consideraron todos los estudios que incluyeran WOM independientemente de si se trataba de WOM o eWOM, considerando que pertenecen a una misma vertiente aunque se potencialicen en medios distintos y con diferentes amplitudes e influencias.

Además, con la finalidad de abarcar gran parte de las expresiones y variaciones sobre el tema, se recopilaron artículos que mencionaban en sus títulos, palabras clave o resúmenes, términos como eWOM, WOM, online reviews, User-Generated Content (UGC), Consumer-Generated Content (CGC), online recommendation, e-satisfaction, e-complaints, online reputation, online travel communities, online opinions, social media marketing, hospitality industry y hotels.

Una vez analizados en profundidad, se identificaron los impactos más recurrentes generados por el eWOM y destacados por los investigadores tanto desde la perspectiva de los consumidores como de la perspectiva de las empresas.

\section{LOS IMPACTOS DEL eWOM}

En la mayoría de los trabajos consultados se identifican los impactos del eWOM desde la perspectiva del consumidor; sin embargo, algunos de ellos analizan en profundidad los impactos desde la perspectiva de la empresa (Figura 1).

Impactos del eWOM desde la perspectiva de los consumidores. 
Cabe señalar que, en gran medida, los estudios consultados pretenden contribuir a las estrategias de marketing de las empresas, dado que analizan el comportamiento del consumidor y sus actitudes hacia ciertas acciones $y$ escenarios. Los temas más destacados hacen referencia al proceso de toma de decisiones, el consumers' drive, la percepción de confianza, el nivel de conocimientos, los comentarios útiles y la intención de compra (Arsal et al., 2010: Black y Kelley, 2009; Dickinger, 2011; Hills y Cairncross, 2009; Kim et al., 2011; Lee et al., 2011; Litvin et al. 2008; Papathanassis y Knolle, 2011; Qu y Lee, 2011; Sparks y Browning, 2011; Vermeulen y Seegers, 2009; Xiang y Gretzel, 2010; Xie et al., 2011; Yacouel y Fleischer, 2011).

Figura 1: Impacto del eWOM desde la perspectiva del consumidor y de la empresa.

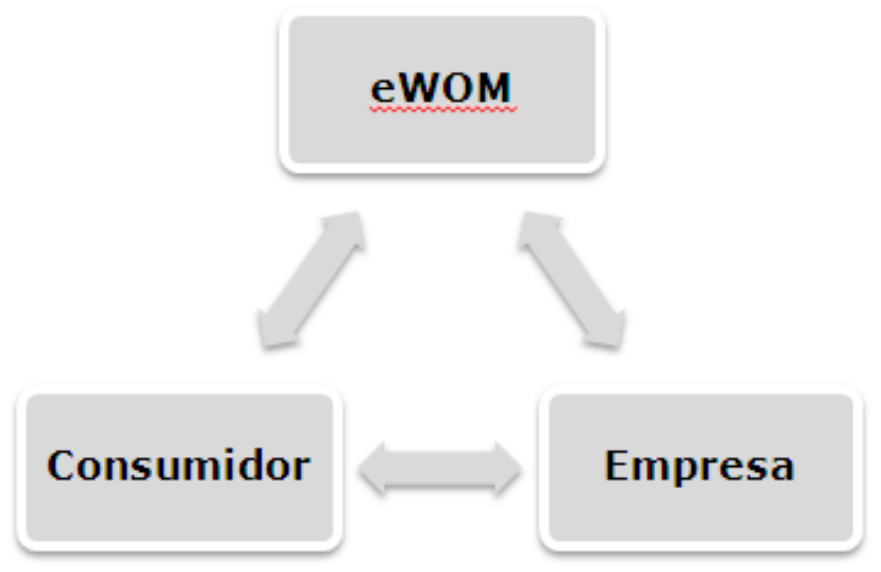

Fuente: elaboración propia.

En mayor o menor medida, todos los estudios consideran la influencia de los comentarios (WOM o eWOM) en el proceso de toma de decisiones. Xie et al (2011) sostienen que el uso del eWOM es frecuente en el mercado hotelero actual y tiene el potencial para influir en la toma de decisiones de los consumidores. Litvin et al. (2008), en la misma línea, señalan que la influencia interpersonal y el Word-of-Mouth (WOM) son la más importante fuente de información cuando un consumidor ejecuta el proceso de decisión de compra. 
Estas influencias son especialmente importantes en la industria turística, cuyos productos intangibles son difíciles de evaluar antes de su consumo.

La literatura académica demuestra que la valencia del eWOM tiene un fuerte impacto en la evaluación de los productos/servicios y las decisiones de compra. Vermeulen y Seegers (2009) argumentan que los comentarios, tanto positivos como negativos, aumentan el conocimiento que los consumidores poseen respecto de los hoteles, mientras que los comentarios positivos, además, mejoran las actitudes hacia los hoteles. Dichos autores sostienen que estos efectos son más fuertes para los hoteles menos conocidos. Su estudio sugiere incluso que las críticas positivas tienen un impacto positivo en el comportamiento del consumidor, mientras que los comentarios negativos tienen poco impacto, aunque esto no significa que los comentarios negativos sean inofensivos.

Por otro lado, Sparks y Browning (2011) mencionan que los consumidores parecen ser más influenciados por la información negativa, especialmente cuando el conjunto total de comentarios son negativos. Sin embargo, un conjunto de información positiva, junto con los detalles de clasificación numérica, aumentan las intenciones de reserva y la confianza del consumidor. Este estudio destaca que la ocurrencia de críticas positivas recientes puede reemplazar o moderar el efecto de un conjunto de críticas negativas anteriores, respecto a la intención de reserva.

Además, variables tales como la confiabilidad, la credibilidad del eWOM, comentarios útiles y el nivel de experiencia (Arsal et al., 2010; Black y Kelley, 2009; Lee et al., 2011; Litvin et al. 2008; Vermeulen y Seegers, 2009; Xie et al., 2011; Yacouel y Fleischer, 2011) se incluyen con frecuencia al analizar el impacto y la influencia de los comentarios desde la perspectiva del consumidor. Estos aspectos, entre otros, contribuyen a la reducción del riesgo percibido (Arsal et al., 2010; Dickinger, 2011; Kim et al., 2011; Sparks y Browning, 2011) durante el proceso de toma de decisión. Papathanassis y Knolle (2011) sostienen que los usuarios participan en actividades de búsqueda de información para minimizar el riesgo asociado a la compra de un paquete donde el producto/servicio es intangible e inseparable. 
Kim et al. (2011) analizaron el comportamiento de género y llegaron a la conclusión de que: "la mujer, por ejemplo, tiene más probabilidad de leer los comentarios por la conveniencia, calidad y por la reducción del riesgo. La utilización de los comentarios online por los hombres depende de su nivel de experiencia". En esta misma línea, Toh et al. (2011) estudiaron los aspectos relacionados con el género, la edad y los ingresos en el comportamiento de compra. Encontraron que las mujeres han superado a los hombres en las actividades de búsqueda de información. También, concluyeron que los consumidores que realizaron reservas online tienden a ser más jóvenes, tener ingresos más altos, y comprar más días de estancia que aquellos que utilizan canales de distribución tradicionales. Por otra parte, las mujeres realizan una búsqueda de información más intensiva acerca de los posibles hoteles y las tarifas que los hombres. La tesis doctoral de Barlés (2010) encontró resultados similares al estudiar el comportamiento de compra de la pareja para el caso de los viajes vacacionales, dándose un proceso de decisión conjunto globalmente pero con mayor peso de la mujer en el caso de la elección de alojamineto.

Otro aspecto relevante identificado en la bibliografía se encuentra relacionado con una mayor facilidad de acceso a la información y el procesamiento de los comentarios (Dickinger, 2011; Papathanassis y Knolle, 2011; Sparks y Browning, 2011; Xiang y Gretzel, 2010). Este factor es importante dada la gran cantidad de información disponible, causante de una compleja tarea de filtrado y análisis de la información por parte de los consumidores. Sparks y Browning (2011) sostienen que hay una gama de factores potenciales que influyen en las decisiones de los consumidores, algunos de ellos engloban el contenido o el objetivo de los comentarios, el tono general o la valencia de los comentarios (como un conjunto de factores), la estructura del conjunto de comentarios (lo que se lee primero) y la facilidad de procesar la información como una generación de clasificaciones numéricas. Además, la facilidad de evaluar la información, así como las clasificaciones generales (p. ej. clasificación hotelera por estrellas o puntuaciones de clientes), pueden tener una mayor influencia sobre las decisiones de compra del producto en comparación con información más detallada. 


\section{Impactos del eWOM desde la perspectiva de las empresas.}

En los estudios analizados se identificaron aspectos relacionados con la gestión de contenidos por parte de las empresas, la posibilidad de hacer un control de calidad de sus productos y servicios y poder interactuar con sus clientes tanto para solucionar eventuales problemas como para hacerles llegar información sobre sus productos. La posibilidad de incrementar las reservas y de generar "Price Premium" son otros temas destacados en las investigaciones (Anderson, 2012; Dickinger, 2011; Hills y Cairncross, 2009; Yacouel y Fleischer, 2011).

Ye et al. (2009) en su estudio demostraron que los comentarios online positivos pueden aumentar significativamente el número de reservas de un hotel, y la varianza o polaridad de los comentarios pueden tener un impacto negativo en la cantidad de ventas online. Los resultados sugieren que una mejora del $10 \%$ en la calificación de los comentarios puede incrementar las ventas en un 4,4\% y un aumento del $10 \%$ en la varianza de los comentarios puede disminuir las ventas en un $2,8 \%$.

En su estudio reciente, Anderson (2012) ha analizado el impacto de las redes sociales en la performance de los hoteles y llegó a las siguientes conclusiones:

1) El porcentaje de consumidores que consultan comentarios en TripAdvisor antes de reservar una habitación de hotel ha ido aumentando con el tiempo, así como el número de comentarios que se leen antes de hacer la elección de hotel.

2) Si un hotel aumenta su puntuación en 1 punto en una escala de 5 puntos (por ejemplo, de 3,3 a 4,3), el hotel puede aumentar su precio en un $11,2 \%$ manteniendo la misma ocupación o cuota de mercado.

3) Un aumento del $1 \%$ en la puntuación de la reputación online del hotel conduce a $0,89 \%$ de incremento en el precio de la tarifa media diaria (ADR). Del mismo modo, este 1\% de aumento en su reputación también conduce a un aumento de ocupación de hasta el 0,54\%. Este 1\% en la 
mejora de la reputación puede generar hasta un 1,42\% de incremento en los ingresos por habitación disponible (RevPAR).

Loureiro y Kastenholz (2011) argumentan que la reputación corporativa juega un papel importante en la percepción del cliente respecto a la capacidad de rendimiento del servicio, lo que conlleva una mayor confianza en la prestación del servicio en la mente del cliente. Jun et al. (2010) agregan que la heterogeneidad de los servicios de alojamiento aumenta la incertidumbre en la toma de decisiones; por lo tanto, las personas se ven obligadas a evaluar diversos atributos y utilizar diferentes fuentes (por ejemplo, páginas web propias de los hoteles, páginas web oficiales de los destinos, portales del opiniones de los clientes). Da Silva y Alwi (2008) añaden que una sólida y positiva reputación corporativa no sólo ayuda a una empresa a lograr una ventaja competitiva, también fomenta la repetición de compra.

Las nuevas pautas del comportamiento del consumidor y las nuevas tecnologías conducen a una mayor transparencia en el mercado (Jun et al., 2010; Toh et al., 2011; Verma, 2010; Wen, 2008) que puede crear oportunidades y al mismo tiempo un mayor riesgo para las empresas. Verma (2010) sostiene que la oferta en el mercado hotelero es cada vez más compleja, debido en gran medida a los avances en la tecnología de la información. Esta situación permite a los clientes comparar y evaluar estratégicamente los costes y beneficios relativos a las diferentes alternativas.

En este sentido, Wen (2008) señala que las estrechas relaciones entre clientes y páginas web de proveedores pueden reducir el peligro de sustitución y ayudar a asegurar una rentabilidad a largo plazo. Enfatiza que la esencia de crear un vínculo con los clientes es conocer cómo hacen sus compras online y qué factores influyen en sus intenciones de compra online.

Otro factor destacado en los estudios se refiere a la lealtad. El término "Lealtad" a menudo se mide por indicadores como la intención de seguir comprando el mismo producto, la intención de comprar más del mismo producto y la repetición de compra (medidas de comportamiento) o deseo de recomendar el producto a otros (indicador actitudinal), reflejando la defensa del producto (Loureiro y Kastenholz, 2011). Callarisa et al. (2012) destacan que 
construir y mantener la lealtad a la marca ha sido un tema central de la teoría de marketing y una práctica en la creación de ventajas competitivas sostenibles.

\section{CONCLUSIONES}

Se observa en este estudio que los impactos del eWOM están teniendo cada vez más relevancia y protagonismo, principalmente en la industria hotelera. Los impactos pueden estructurarse en dos líneas principales: el impacto desde la perspectiva del consumidor y el impacto desde la perspectiva de la empresa.

Los principales impactos identificados desde la perspectiva del consumidor se encuentran relacionados con aspectos relativos al proceso de decisión de compra, a la aceptación del producto, a la reducción del riesgo en la compra, a la percepción de credibilidad/confianza, a la comparación entre diferentes hoteles, al conocimiento de marca, a la intención de compra y a la lealtad. En general, estos aspectos contribuyen en las decisiones de los consumidores y pueden verse afectados tanto positivamente como negativamente.

Por otra parte, los impactos desde la perspectiva de las empresas, pueden ser considerados como una amenaza o como una oportunidad y ventaja competitiva. Entre ellos, los principales aspectos identificados son: el control de calidad y puesta en marcha de nuevos procedimientos, posibilidad de ofertar precios más altos "Price Premium", interacción con los consumidores y posibilidad de respuesta para la solución de eventuales problemas, estrategias de marketing específicas para los targets y sus necesidades generando lealtad por parte de los clientes, comparación de la reputación online con la competencia, entre otros aspectos que cada día las nuevas tecnologías introducen en el mercado. 
La influencia del eWOM es un tema que gana cada vez más relevancia principalmente en la industria hotelera. Todavía se están desarrollando estudios relacionados con los impactos que esta variable tiene en el comportamiento de los consumidores y cómo las empresas deben afrontar esta nueva realidad. La gestión del eWOM se presenta como una nueva necesidad y puede convertirse en una ventaja competitiva importante para la supervivencia de las empresas en el mercado.

\section{BIBLIOGRAFÍA}

Anderson, C. K. (2012). The Impact of Social Media on Lodging Performance. Cornell Hospitality Report, 12(15).

Arsal, I., Woosnam, K. M., Baldwin, E. D. \& Backman, S. J. (2010). Residents as Travel Destination Information Providers: An Online Community Perspective. Journal of Travel Research, 49(4), pp. 400-413.

Barlés, M. J. (2010). La toma de decisiones familiares de compra, el conflicto y las tácticas de influencia en la pareja. El papel de la mujer en las decisiones vacacionales. Tesis doctoral. Universidad de Zaragoza.

Black, H. G. \& Kelley, S. W. (2009). A Storytelling Perspective on Online Customer Reviews Reporting Service Failure and Recovery. Journal of Travel \& Tourism Marketing, 26, pp. 169-179.

Callarisa, L., García, J. S., Cardiff, J. \& Roshchina, A. (2012). Harnessing social media platforms to measure customer-based hotel brand equity. Tourism Management Perspectives, 4, pp. 73-79.

Casaló, L., Flavián, C. \& Guinalíu, M. (2010). Determinants of the intention to participate in firm-hosted online travel communities and effects on consumer behavioral intentions. Tourism Management, 31, pp. 898-911.

Da Silva, R. V. \& Alwi, S .F. S. (2008). Online corporate brand image, satisfaction and loyalty. Brand Management, 16(3), pp. 119-144. 
Dickinger, A. \& Basu, K. (1994). Customer loyalty: toward an integrated conceptual framework. Academy of Marketing Science, 22(2), pp. 99113.

Dickinger, A. (2011). The Trustworthiness of Online Channels for Experienceand Goal-Directed Search Tasks. Journal of Travel Research, 50(4), pp. 378-391.

Harrison-Wallker, L. J. (2001). The measurement of word of mouth communication and an investigation of service quality and customer commitment as potential antecedents. Journal of Service Research, 4(1), pp. 60-75.

Hills, J. R. \& Cairncross, G. (2009). Small accommodation providers and UGC web sites: perceptions and practices. International Journal of Contemporary Hospitality Management, 23(1), pp. 26-43.

Jun, S. H., Vogt, C. A. \& MacKay, K. J. (2010). Online Information Search Strategies: A Focus On Flights and Accommodation. Journal of Travel \& Tourism Marketing, 27, pp. 579-595.

Kim, E. E. K., Mattila, A. S. \& Baloglu, S. (2011). Effects of Gender and Expertise on Consumers' Motivation to Read Online Hotel Reviews. Cornell Hospitality Quarterly, 52(4), pp. 399-406.

Lee, H. A., Law, R. \& Murphy, J. (2011). Helpful Reviewers in TripAdvisor, an Online Travel Community. Journal of Travel \& Tourism Marketing, 28, pp. 675-688.

Litvin, S. W., Goldsmith, R. E. \& Pan, B. (2008). Electronic word-of-mouth in hospitality and tourism management. Tourism Management, 29, pp. 458468.

Loureiro, S. M. C. \& Kastenholz, E. (2011). Corporate reputation, satisfaction, delight, and loyalty towards rural lodging units in Portugal. International Journal of Hospitality Management, 30, pp. 575-583.

Papathanassis, A. \& Knolle, F. (2011). Exploring the adoption and processing of online holiday reviews: A grounded theory approach. Tourism Management, 32, pp. 215-224. 
Qu, H. \& Lee, H. (2011). Travelers' social identification and membership behaviors in online travel community. Tourism Management, 32, pp. 1262-1270.

Schiffman, L. G. \& Kanuk, L. L. (2000). Consumer Behavior, 7th edition. Upper Saddle River, NJ: Prentice Hall.

Sparks, B. A. \& Browning, V. (2011). The impact of online reviews on hotel booking intentions and perception of trust. Tourism Management, 32, pp. 1310-1323.

Sun, L. B. \& Qu, H. (2011). Is There Any Gender effect on the Relationship Between Service Quality and Word-of-Mouth? Journal of Travel \& Tourism Marketing, 28, pp. 210-224.

Toh, R. S., DeKay, C. F. \& Raven, P. (2011). Travel Planning: Searching for and Booking Hotels on the Internet. Cornell Hospitality Quarterly, 52(4), pp. 388-398.

Verma, R. (2010). Customer Choice Modeling in Hospitality Services: A Review of Past Research and Discussion of Some New Applications. Cornell Hospitality Quarterly, 51(4), pp. 470-478.

Vermeulen, I. E. \& Seegers, D. (2009). Tried and tested: The impact of online hotel reviews on consumer consideration. Tourism Management, 30, pp. 123-127.

Wen, I. (2008). Factors affecting the online travel buying decision: a review. International Journal of Contemporary Hospitality Management, 21(6), pp. 752-765.

Worthington, S., Russell-Bennett, R. \& Hartel, C. (2010). A tri-dimensional approach for auditing brand loyalty. Brand Management, 17, pp. 243253.

Xiang, Z. \& Gretzel, U. (2010). Role of social media in online travel information search. Tourism Management, 31, pp. 179-188.

Xie, H. J., Miao, L., Kuo, P. \& Lee, B. (2011). Consumers' responses to ambivalent online hotel reviews: The role of perceived source credibility and predecisional disposition. International Journal of Hospitality Management, 30, pp. 178-183. 
Yacouel, N. \& Fleischer, A. (2011). The Role of Cybermediaries in Reputation Building and Price Premiums in the Online Hotel Market. Journal of Travel Research, 50(1), pp. 1-8.

Ye, Q., Law, R. \& Gu, B. (2009). The impact of online user reviews on hotel room sales. International Journal of Hospitality Management, 28, pp. 180-182.

Yoon, Y. \& Uysal, M. (2005). An examination of the effects of motivation and satisfaction on destination loyalty: a structural model. Tourism Management, 26(1), pp. 45-56. 